\title{
Synthesis of Fused-Oxazines from Cyclic Ketoximes Via $\alpha$-Nitrosoalkenes
}

\author{
SANTOSH L. GAONKAR ${ }^{1,2 *}$ and K. M. LOKANATHA RAI ${ }^{2}$ \\ 1Department of Chemistry, Manipal Institute of Technology, \\ Manipal University, Manipal-576104, India. \\ 2Department of Studies in Chemistry, University of Mysore, \\ Manasagangotri, Mysore-570 006, India. \\ ${ }^{*}$ Corresponding author E-mail: gaonkarslg@ rediffmail.com \\ http://dx.doi.org/10.13005/ojc/330238
}

(Received: March 03, 2017; Accepted: April 04, 2017)

\begin{abstract}
Cyclic ketoximes having a $\alpha$-methylene group on reaction with chloramine-T followed by treatment with triethylamine generate $\alpha$-nitrosoalkenes via $\alpha$-chloronitroso intermediates, which are further treated with alkenes to give fused-oxazines.
\end{abstract}

Keywords: Fused-oxazines, $\alpha$-nitrosoalkenes, Chloramine-T.

\section{INTRODUCTION}

1,2-Oxazine and their fused counterparts received interest in these years due to their diverse synthetic and pharmacological importance. 1,2Oxazine derivatives are useful synthetic building blocks in heterocyclic chemistry. In our preceding report $^{1}$, we have disclosed the method to obtain $\alpha$-nitrosoalkenes from ketoximes having $\alpha$-methylene group and their cycloaddition with olefinic compounds to 1,2-oxazines. $\alpha$-Nitrosoalkenes behave like dienes (4ח-elecron component) in Hetero-Diels-Alder reaction. Further to our research on oxazines and their counterparts, we are reporting the synthesis of $\alpha$-nitrosoalkenes from cyclic ketoximes bearing a $\alpha$-methylene group and their cycloaddition with dienophiles to yield fused-oxazines, which are having huge synthetic applications ${ }^{2,3}$ and biological use as therapeutic agents ${ }^{4-7}$. Generation of $\alpha$-nitrosoalkenes is quite difficult. Usually they are reactive intermediates and unstable, hence they are only generated in situr.

In our prior work chloramine-T was used extensively for the synthesis of $\alpha$-nitrosoolefins ${ }^{1}$, azoalkenes ${ }^{8-9}$, nitrile oxides ${ }^{10}$, nitrile imines ${ }^{11}$ etc., which undergo cycloaddition with active dienes yield bioactive heterocycles. During the studies, cyclohexanone oxime was reacted with chloramine-T formed a blue colour indicates the 
formation of chloro-nitroso intermediate, which produces 1-nitroso-cyclohexene on treatment with a base. With this achievement, we are now reporting a new method for the conversion of cyclic ketoximes heaving an active methylene group into $\alpha$-chloronitroso intermediates, which are appropriate for in situ formation of $\alpha$-nitrosoalkenes which acts as dienes and undergo [4+2] cycloaddition with dienophiles.

\section{RESULTS AND DISCUSSIONS}

Cyclic ketoximes having a $\alpha$-hydrogen atom on treatment with chloramine-T followed by treatment with a base are oxidized to $\alpha$-nitroso alkenes which undergo Hetero-Diels-Alder reaction with olefinic compounds to produce fused-oxazines in good yield. Typically, the reaction was performed by boiling an equimolar mixture of cyclic ketoxime and chloramine-T in ethyl alcohol followed by addition of a base and an alkene. Overall fused-1,2-oxazines are synthesized in good yield as indicated in the scheme 1.

${ }^{1} \mathrm{HNMR},{ }^{13} \mathrm{CNMR}$, Mass spectral analyses and elemental analyses established the structure of the fused-oxazines synthesized. The cycloaddition reaction was regioselective. Proton NMR spectrum of fused-oxazine 4 (when $\mathrm{X}=\mathrm{H}$ ) showed dd around $\delta$ 3.6-4.4 ppm which is due to $\mathrm{H}_{3}$ and triplet in the region $\delta 1.70-1.90 \mathrm{ppm}$ is the signal for $\mathrm{H}_{4}$. Where as in fused-oxazine 4 (when $\mathrm{X}=\mathrm{CH}_{3}$ ), no signal observed for $\mathrm{H}_{3}$ in the above said region, while a new signal was appeared at $\delta 1.69 \mathrm{ppm}$ designates methyl group and $\mathrm{H}_{4}$ observed in the region $\delta 1.70-1.90 \mathrm{ppm}$ as doublet.

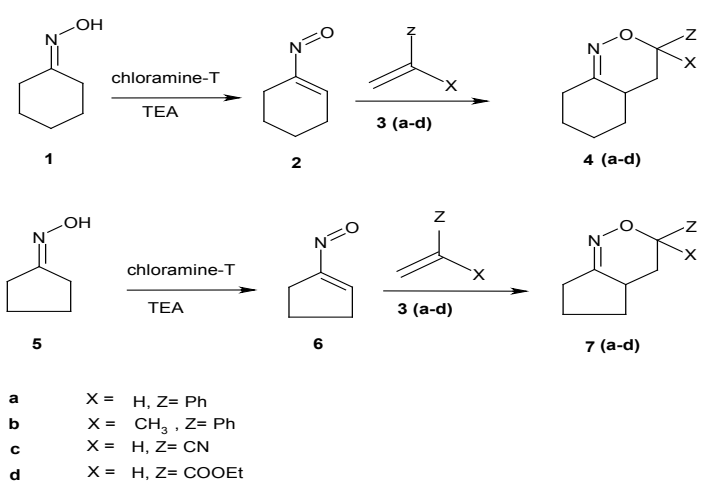

Scheme 1
${ }^{13} \mathrm{C}$ NMR spectrum of all fused-oxazines gave expected signals for the newly formed ring carbons. For instance, in fused-oxazine 4 (when $\mathrm{X}=\mathrm{H}$ ) the peak due to $\mathrm{C}_{3}$ observed around $\delta 54-67$ ppm while 4 (when $\mathrm{X}=\mathrm{CH}_{3}$ ) seen around $\delta 62-69 \mathrm{ppm}$ and a new signal was observed around $\delta 23-26 \mathrm{ppm}$ indicative of the methyl group. The signal in oxazine $4 \mathrm{c}$ at $\delta 119 \mathrm{ppm}$ is indicative of the $\mathrm{CN}$ group. The stable molecular ion peaks were seen in the Mass spectrum which supports the structure of the newly formed oxazines. The product formation further confirmed by elemental analyses. 1,2-Oxazines and their fused counterparts are potentially very useful heterocycles in synthetic chemistry, and act as useful building blocks for the construction of complex and new heterocyclic compounds.

In summary we have established that fusedoxazines can be produced by the reaction of cyclic ketoximes having a $\alpha$-methylene group with olefinic compounds in the presence of chloramines- $T$ and a base in good yield.

\section{EXPERIMENTAL}

${ }^{1} \mathrm{H}$ NMR and ${ }^{13} \mathrm{C}$ NMR spectra were taken at $400 \mathrm{MHz}$ and $100 \mathrm{MHz}$ respectively on Bruker Avance 400 spectrometer using DMSO- $\mathrm{d}_{6}$ or $\mathrm{CDCl}_{3}$ as solvents and TMS as internal standard. The chemical shifts are stated in $\delta$ ppm downfield shift from TMS. Mass spectrum was recorded on a Finnigan 4021 mass spectrometer using ionizing energy of $35 \mathrm{ev}$. Elemental analyses were performed using Vario-EL elemental analyzer. Reactions were checked for the completion by thin layer chromatography (TLC) on precoated silica gel plates using chloroform-ethyl acetate $(7: 3)$ as eluent.

Representative process for the preparation of 3-Phenyl-4,4a,5,6,7,8-hexahydro-3H-benzo[c] $[1,2]$ oxazine $4 a$

A mixture of cyclohexanone oxime 1 (1.0 $\mathrm{g}, 8.85 \mathrm{mmol})$ and chloramine-T. $3 \mathrm{H}_{2} \mathrm{O}(2.51 \mathrm{~g}, 8.93$ $\mathrm{mmol})$ in ethyl alcohol $(6 \mathrm{ml})$ were boiled for 1 hour. The reaction mass was cooled to $\mathrm{rt}$. and triethylamine $(1 \mathrm{ml})$ was added. The reaction mixture was further stirred for 30 minutes at rt. $3 a(0.94 \mathrm{~g}, 9.03 \mathrm{mmol})$ dissolved in ethyl alcohol $(3 \mathrm{ml})$ was then added to the above reaction mass and was further boiled 2 $\mathrm{hr}$. After the completion of the reaction, ethyl alcohol 
was removed under vacuum and the residue left behind was extracted with chloroform (2X20 ml). The combined chloroform extract was washed with $\mathrm{H}_{2} \mathrm{O}(15 \mathrm{ml})$, with dilute $\mathrm{NaOH}$ solution $(210 \mathrm{ml})$ and dried over anhydrous magnesium sulfate. Chloroform was removed under vacuum and the oily mass left behind was purified by column chromatography $\left(\mathrm{CHCl}_{3}\right.$ :EtOAc, 8:2) to give $4 \mathrm{a}$ as a pale yellow oily product to yield $1.25 \mathrm{~g} \mathrm{(66 \% );}{ }^{1} \mathrm{HNMR}\left(\mathrm{CDCl}_{3}, 400\right.$ $\mathrm{MHz}): \delta 1.24-1.31\left(\mathrm{~m}, 4 \mathrm{H}, 2 \mathrm{X}-\mathrm{CH}_{2}\right), 1.45-1.55$ $\left(\mathrm{m}, 5 \mathrm{H}, 2 \mathrm{X}-\mathrm{CH}_{2}\right.$ and $\left.-\mathrm{CH}\right), 1.89\left(\mathrm{t}, 2 \mathrm{H},-\mathrm{CH}_{2}\right), 4.61$ (dd, $1 \mathrm{H}, \mathrm{J}=9.7 \mathrm{~Hz} \& 2.5 \mathrm{~Hz},-\mathrm{CH}$ ), 7.31 (s, 5H, ArH); ${ }^{13} \mathrm{C} \mathrm{NMR}\left(\mathrm{CDCl}_{3}\right): \delta 24.4,26.8,29.5,30.9,32.6$, 35.4, 81.2, 125.1, 128.4, 141.9, 162.5; MS (relative abundance) m/z: $215\left(\mathrm{M}^{+}, 8 \%\right), 138,120,95$ (100\%), 77. $\mathrm{C}, \mathrm{H}$ and $\mathrm{N}$. Calcd. for $\mathrm{C}_{14} \mathrm{H}_{17} \mathrm{NO}$ : C, 78.10; $\mathrm{H}, 7.96$; N, $6.51 \%$. Found: C, 78.19; H, 8.04; N, $6.43 \%$.

\section{3-Methyl-3-phenyl-4,4a,5,6,7,8-hexahydro-3H- benzo[c][1,2]oxazine $4 b$}

The product was synthesized from 1 $(1.0 \mathrm{~g}, 8.85 \mathrm{mmol}), 3 \mathrm{~b}(1.06 \mathrm{~g}, 8.98 \mathrm{mmol})$, chloramineT.3 $\mathrm{H}_{2} \mathrm{O}(2.51 \mathrm{~g}, 8.93 \mathrm{mmol})$ and TEA to give yellow oily product to yield $1.31 \mathrm{~g}(65 \%) ;{ }^{1} \mathrm{H} \mathrm{NMR} \mathrm{CDCl}_{3}$ : $\delta$ 1.26-1.35 (m, 4H, 2XCH $), 1.45-1.55(\mathrm{~m}, 5 \mathrm{H}$, $2 \mathrm{XCH}_{2}$ and $\left.\mathrm{CH}\right), 1.71\left(\mathrm{~s}, 3 \mathrm{H}, \mathrm{CH}_{3}\right), 1.93(\mathrm{~d}, 2 \mathrm{H}$, $\left.\mathrm{CH}_{2}\right), 7.41$ (s, 5H, ArH); ${ }^{13} \mathrm{C} \mathrm{NMR}\left(\mathrm{CDCl}_{3}\right): \delta 23.6$,<smiles>CC1(c2ccccc2)C[C@H]2CCCC[C@@]2(Br)C1=NO</smiles>

\section{4b}<smiles>CC1CCC2CC(C#N)ON=C2C1</smiles>

$26.1,29.2,30.7,31.5,32.9,34.2,74.6,125.9$, 128.5, 129.0, 139.1, 162.2; MS (relative abundance) m/z: $229\left(\mathrm{M}^{+}, 12 \%\right), 152,134,95$ (100\%), 77. C, H and N. Calcd. for $\mathrm{C}_{15} \mathrm{H}_{19} \mathrm{NO}: \mathrm{C}, 78.56 ; \mathrm{H}, 8.35 ; \mathrm{N}$, $6.11 \%$. Found: C, 78.50; H, 8.41; N, 6.03\%.

\section{4,4a,5,6,7,8-Hexahydro-3H-benzo[c][1,2]oxazine- 3-carbonitrile 4c}

The product was synthesized from 1 $(1.0 \mathrm{~g}, 8.85 \mathrm{mmol}), 3 \mathrm{c}(0.48 \mathrm{~g}, 9.05 \mathrm{mmol})$, chloramineT. $3 \mathrm{H}_{2} \mathrm{O}(2.51 \mathrm{~g}, 8.93 \mathrm{mmol})$ and TEA as a yellow oily product to yield $1.03 \mathrm{~g}(71 \%) ;{ }^{1} \mathrm{H} \mathrm{NMR} \mathrm{CDCl}_{3}$ : 61.28-1.34 (m, $\left.4 \mathrm{H}, 2 \mathrm{XCH}_{2}\right), 1.42-1.51\left(\mathrm{~m}, 5 \mathrm{H}, 2 \mathrm{XCH}_{2}\right.$ and $\mathrm{CH}), 2.12-2.17\left(\mathrm{~m}, 2 \mathrm{H}, \mathrm{CH}_{2}\right), 4.69(\mathrm{dd}, 1 \mathrm{H}, \mathrm{CH})$; ${ }^{13} \mathrm{C} \mathrm{NMR}\left(\mathrm{CDCl}_{3}\right): \delta \mathrm{d} 23.2,26.1,30.9,31.3,34.3$, 35.5, 70.3, 118.2, 162.2; MS (relative abundance) $\mathrm{m} / \mathrm{z}: 164\left(\mathrm{M}^{+}, 13 \%\right), 163,136,95(100 \%), 69 . \mathrm{C}$, $\mathrm{H}$ and N. Calcd. for $\mathrm{C}_{9} \mathrm{H}_{12} \mathrm{~N}_{2} \mathrm{O}: \mathrm{C}, 65.83 ; \mathrm{H}, 7.37 ; \mathrm{N}$, $17.06 \%$. Found: C, 65.89; H, 7.30; N, $17.11 \%$.

\section{4,4a,5,6,7,8-Hexahydro-3H-benzo[c][1,2]oxazine- 3-carboxylic acid ethyl ester $4 d$}

The product was synthesized from 1 $(1.0 \mathrm{~g}, 8.85 \mathrm{mmol}), 3 \mathrm{~d}(0.90 \mathrm{~g}, 9.0 \mathrm{mmol})$, chloramineT. $3 \mathrm{H}_{2} \mathrm{O}(2.51 \mathrm{~g}, 8.93 \mathrm{mmol})$ and TEA as a pale yellow oily product to yield $0.48 \mathrm{~g}(72 \%) ;{ }^{1} \mathrm{H} \mathrm{NMR} \mathrm{CDCl}_{3}$ : $\delta 1.29\left(\mathrm{t}, 3 \mathrm{H}, \mathrm{CH}_{3}\right), 1.35-1.44\left(\mathrm{~m}, 8 \mathrm{H}, \mathrm{CH}_{2}\right), 1.55-161$ $(\mathrm{m}, 1 \mathrm{H}, \mathrm{CH}), 2.22\left(\mathrm{t}, 2 \mathrm{H}, \mathrm{CH}_{2}\right), 4.28(\mathrm{dd}, 1 \mathrm{H}, \mathrm{CH})$, 4.69 (q, $\left.2 \mathrm{H}, \mathrm{CH}_{2}\right) ;{ }^{13} \mathrm{C} \mathrm{NMR} \mathrm{CDCl}_{3}: \delta 12.6,27.3,29.4$, 30.8, 33.0, 35.3, 61.1, 83.2, 163.2, 174.3; MS (relative abundance) m/z: $211\left(\mathrm{M}^{+}, 22 \%\right), 166(100 \%), 138$, 95, 71. C, $\mathrm{H}$ and N. Calcd. for $\mathrm{C}_{11} \mathrm{H}_{17} \mathrm{NO}_{3}: \mathrm{C}, 62.54$; $\mathrm{H}, 8.11 ; \mathrm{N}, 6.63 \%$. Found: C, 62.48; H, 8.19; N, $6.56 \%$.

\section{3-Phenyl-3,4,4a,5,6,7-hexahydro-cyclopenta[c] $[1,2]$ oxazine $7 \mathrm{a}$}

The product was synthesized from $5(1.0 \mathrm{~g}$, $10.10 \mathrm{mmol}), 3 \mathrm{a}(1.07 \mathrm{~g}, 10.28 \mathrm{mmol})$, chloramineT. $3 \mathrm{H}_{2} \mathrm{O}(2.85 \mathrm{~g}, 10.15 \mathrm{mmol})$ and TEA to give a pale yellow oily product to yield $0.48 \mathrm{~g}(64 \%) ;{ }^{1} \mathrm{H}$ NMR $\mathrm{CDCl}_{3}: \delta 1.33\left(\mathrm{t}, 2 \mathrm{H}, \mathrm{CH}_{2}\right), 1.46-1.55\left(\mathrm{~m}, 5 \mathrm{H}, 2 \mathrm{XCH}_{2}\right.$ and $\mathrm{CH}$ ), $2.01\left(\mathrm{t}, 2 \mathrm{H}, \mathrm{CH}_{2}\right), 4.42(\mathrm{dd}, 1 \mathrm{H}, \mathrm{CH}), 7.39$ (s, 5H, ArH); ${ }^{13} \mathrm{C} \mathrm{NMR} \mathrm{CDCl}: \delta 26.2,27.9,32.6,36.5$, 41.2, 78.4, 125.8, 128.9, 129.2, 140.1, 161.0; MS (relative abundance) $\mathrm{m} / \mathrm{z}: 201\left(\mathrm{M}^{+}, 11 \%\right), 124,120$, $81(100 \%), 77$. C, $\mathrm{H}$ and N. Calcd. for $\mathrm{C}_{13} \mathrm{H}_{15} \mathrm{NO}: \mathrm{C}$, 77.58 ; H, 7.51; N, $6.96 \%$. Found: C 77.48; H, 7.59; $\mathrm{N}, 6.91 \%$. 
3-Methyl-3-phenyl-3,4,4a,5,6,7-hexahydrocyclopenta[c][1,2]oxazine $7 \mathrm{~b}$

The product was synthesized from $5(1.0 \mathrm{~g}$, $10.1 \mathrm{mmol}), 3 \mathrm{~b}(1.20 \mathrm{~g}, 10.16 \mathrm{mmol})$, chloramine-T.3 $\mathrm{H}_{2} \mathrm{O}(2.85 \mathrm{~g}, 10.15 \mathrm{mmol})$ and TEA to give yellow oily product to yield $0.48 \mathrm{~g}(67 \%) ;{ }^{1} \mathrm{H} \mathrm{NMR} \mathrm{CDCl}_{3}: \delta 1.34$ $\left(\mathrm{t}, 2 \mathrm{H}, \mathrm{CH}_{2}\right), 1.48-1.55\left(\mathrm{~m}, 4 \mathrm{H}, 2 \mathrm{XCH}_{2}\right), 1.64(\mathrm{~s}, 3 \mathrm{H}$, $\left.\mathrm{CH}_{3}\right), 1.64-1.68(\mathrm{~m}, 1 \mathrm{H}, \mathrm{CH}), 1.98\left(\mathrm{~d}, 2 \mathrm{H}, \mathrm{CH}_{2}\right), 7.35$ (s, $5 \mathrm{H}, \mathrm{ArH}) ;{ }^{13} \mathrm{C} \mathrm{NMR} \mathrm{CDCl}: \delta 224.2,26.4,33.3,36.3$, 38.9, 79.2, 125.3, 128.4, 128.8, 140.7, 162.3; MS (relative abundance) $\mathrm{m} / \mathrm{z}: 215\left(\mathrm{M}^{+}, 9 \%\right), 138,134$, $81(100 \%), 77 . \mathrm{C}, \mathrm{H}$ and N. Calcd. for $\mathrm{C}_{14} \mathrm{H}_{17} \mathrm{NO}: \mathrm{C}$, 78.10; $\mathrm{H}, 7.96 ; \mathrm{N}, 6.51 \%$. Found: $\mathrm{C}, 78.19 ; \mathrm{H}, 7.90$; $\mathrm{N}, 6.58 \%$.

\section{3,4,4a,5,6,7-hexahydro-cyclopenta[c][1,2] oxazine-3-carbonitrile 7c}

The product was synthesized from $5(1.0 \mathrm{~g}$, $10.1 \mathrm{mmol}), 3 \mathrm{c}(0.55 \mathrm{~g}, 10.37 \mathrm{mmol})$, chloramine-T.3 $\mathrm{H}_{2} \mathrm{O}(2.85 \mathrm{~g}, 10.15 \mathrm{mmol})$ and TEA as pale brown oily product to yield $0.48 \mathrm{~g}(69 \%) ;{ }^{1} \mathrm{H} \mathrm{NMR} \mathrm{CDCl}_{3}$ : $\delta 1.39\left(\mathrm{t}, 3 \mathrm{H}, \mathrm{CH}_{2}\right), 1.51-1.60\left(\mathrm{~m}, 5 \mathrm{H}, 2 \mathrm{XCH}_{2}\right.$ and $\left.\mathrm{CH}\right)$, $1.99\left(\mathrm{t}, 2 \mathrm{H}, \mathrm{CH}_{2}\right), 4.79$ (dd, $\left.1 \mathrm{H}, \mathrm{CH}\right) ;{ }^{13} \mathrm{C} \mathrm{NMR} \mathrm{CDCl}_{3}$ : $\delta$ 22.2, 27.8, 32.9, 37.6, 41.1, 71.2, 118.3, 161.5; MS (relative abundance) $\mathrm{m} / \mathrm{z}: 150\left(\mathrm{M}^{+}, 10 \%\right), 149$, 122, 81 (100\%), 69. C, $\mathrm{H}$ and N. Calcd. for $\mathrm{C}_{8} \mathrm{H}_{10} \mathrm{~N}_{2} \mathrm{O}$ :
C, 63.98; H, 6.71; N, 18.65 \%. Found: C, 63.90; $\mathrm{H}, 6.77 ; \mathrm{N}, 18.69 \%$.

2-Pheny $-3,4,4 \mathrm{a}, 5,6,7-\mathrm{hexahydro}-2 \mathrm{H}-$ cyclopenta[c]pyridazine-3-carboxylic acid ethyl ester $7 d$

The product was synthesized from $5(1.0 \mathrm{~g}$, $10.1 \mathrm{mmol}$ ), $3 \mathrm{~d}(1.03 \mathrm{~g}, 10.30 \mathrm{mmol}$ ), chloramine-T.3 $\mathrm{H}_{2} \mathrm{O}(2.85 \mathrm{~g}, 10.15 \mathrm{mmol})$ and TEA as a yellow oily mass to yield $0.48 \mathrm{~g}(68 \%) ;{ }^{1} \mathrm{H}$ NMR $\mathrm{CDCl}_{3}: \delta 1.29$ $\left(\mathrm{t}, 3 \mathrm{H}, \mathrm{CH}_{3}\right), 1.39-1.52\left(\mathrm{~m}, 6 \mathrm{H}, 3 \mathrm{XCH}_{2}\right), 1.55-1.63$ $(\mathrm{m}, 1 \mathrm{H}, \mathrm{CH}), 1.954-2.01\left(\mathrm{~m}, 2 \mathrm{H}, \mathrm{CH}_{2}\right), 4.29(\mathrm{dd}, 1 \mathrm{H}$, $\mathrm{CH}), 4.73\left(\mathrm{q}, 2 \mathrm{H}, \mathrm{CH}_{2}\right) ;{ }^{13} \mathrm{C} \mathrm{NMR} \mathrm{CDCl}:$ : $\delta 14.3,21.3$, 26.2, 30.8, 31.2, 33.9, 35.6, 61.2, 84.2, 161.2, 173.6; MS (relative abundance) m/z: $197\left(\mathrm{M}^{+}, 11 \%\right), 197$, $152(100 \%), 124,116,81,71 . \mathrm{C}, \mathrm{H}$ and N. Calcd. for $\mathrm{C}_{10} \mathrm{H}_{15} \mathrm{NO}_{3}: \mathrm{C}, 60.90 ; \mathrm{H}, 7.67 ; \mathrm{N}, 7.10 \%$. Found: $\mathrm{C}, 60.99 ; \mathrm{H}, 7.60 ; \mathrm{N}, 7.06 \%$.

\section{CONCLUSION}

We have established a simple and effective method for the synthesis of fused-oxazines from cyclic ketoximes by Heter-Diels-Alder cycloaddition of in situ generated $\alpha$-nitroso olefins with various alkenes.

\section{REFERENCES}

1. Gaonkar, S. L.; Rai, K. M. L. J. Heterocyclic Chem. 2005, 42, 877.

2. Faragher, R.; Gilchrist, T. L. J. Chem. Soc. 7. Perkin Trans. I , 1979, 249.

3. Ito, S.; Saito, I. Tetrahedron Lett. 2005, 46 , 5969.

4. Polanc, S. J. Heterocyclic Chem. 2005, 42, 401.

5. Liyebris, C.; Martinsson, J.; Williams, L. T. M.; Barker, E.; James, E. S.; Duffy; Nygren A.; James, S. Bioorg. Med.Chem. 2002, 10, 3197.
6. Bekhit, A. A. Boll Chim Farm. 2001, 140, 243.

Osborn H. M. I.; Coisson, D. Mini-Rev. Org. Chem. 2004, 1, 41.

8. Gaonkar, S. L.; Rai, K. M. L. Tetrahedron Lett. 2005, 46, 5969.

9. Gaonkar, S. L.; Rai, K. M. L. J. Heterocyclic Chem. 2015, 52, 1346.

10. Gaonkar, S. L.; Rai, K. M. L.; Prabhuswamy, B. Med. Chem. Res. 2007, 15, 407.

11. Gaonkar, S. L.; Rai, K. M. L.; Prabhuswamy, Eur. J. Med. Chem. 2006, 41, 841. 